\title{
Aprendendo, ensinando e mapeando território: vivências de acadêmicos de enfermagem
}

\author{
Learning, teaching and mapping territory: experiences of nursing academics
}

Aprendiendo, enseñando y mapeando territorio: vivencias de académicos de enfermería

Katia Fernanda Alves Moreira ${ }^{1}$, Daiana Evangelista Rodrigues ${ }^{1}$, Lerissa Nauana Ferreira ${ }^{{ }^{*}}$, Marcos Antônio Sales Rodrigues ${ }^{1}$, Davisson Michetti de Oliveira², Priscilla Perez da Silva Pereira ${ }^{1}$.

\section{RESUMO}

Objetivo: Este artigo teve como objetivo descrever a experiencia de discentes e docentes sobre o conhecimento do território de uma equipe da Estratégia de Saúde da Família (ESF) no município de Porto Velho, Rondônia. Método: Para o diagnóstico situacional utilizou-se a técnica de Territorialização, além de dados secundários do e-SUS AB. Para a confecção do mapa do território utilizou-se o método Círculos de Cultura. Resultados: Os Círculos de Cultura possibilitaram a descoberta dos estrangulamentos, nós críticos, nas perspectivas e necessidades da comunidade. Os alunos participaram ativamente da territorialização, conhecendo a dinâmica da realidade do território, estreitando os laços com a equipe particularmente com os ACS e interagindo com os usuários. Conclusão: A experiência mostrou o papel fundamental do enfermeiro no processo de territorialização e no desenvolvimento de ações de promoção à saúde, permitindo aos acadêmicos de enfermagem o desenvolvimento de olhares críticos e voltados para os problemas reais da população, dotando-os das competências e habilidades para o trabalho no Sistema Único de Saúde e na Atenção Primária à Saúde.

Palavras-chave: Atenção Primária à Saúde, Educação Permanente, Enfermagem em Saúde Pública.

\begin{abstract}
Objective: This article aimed to report the experience of students and teachers about the knowledge of the territory of a team of the Family Health Strategy (ESF) in the city of Porto Velho, Rondônia. Method: For the situational diagnosis the technique of Territorialization was used, in addition to secondary data of e-SUS AB. The Circles of Culture method was used for the mapping of the territory. Results: The Culture Circles enabled the discovery of bottlenecks, critical nodes, in the perspectives and needs of the community. The students participated actively in the territorialization, knowing the dynamics of the reality of the territory, narrowing the bonds with the team particularly with the ACS and interacting with the users. Conclusion: Experience has shown the fundamental role of nurses in the process of territorialization and in the development of actions to promote health, allowing nursing students to develop critical looks focused on the real problems of the population, equipping them with the skills and abilities for work in the Unified Health System and in Primary Health Care.
\end{abstract}

Keywords: Primary Health Care, Permanent Education, Nursing in Public Health.

\footnotetext{
1 Fundação Universidade Federal de Rondônia (UNIR). Porto Velho-RO. *E-mail: lerissaferreira@gmail.com

2 Hospital Sofia Feldman (HSF). Belo Horizonte-MG.
}

SUBMETIDO EM: 10/2018

ACEITO EM: 11/2018

PUBLICADO EM: 01/2019 


\section{RESUMEN}

Objetivo: Este artículo tuvo como objetivo relatar la experiencia de discentes y docentes sobre el conocimiento del territorio de un equipo de la Estrategia de Salud de la Familia (ESF) en el municipio de Porto Velho, Rondônia. Método: Para el diagnóstico situacional se utilizó la técnica de Territorialización, además de datos secundarios del e-SUS AB. Para la confección del mapa del territorio se utilizó el método Círculos de Cultura. Resultados: Los Círculos de Cultura posibilitar el descubrimiento de los estrangulamientos, los críticos, en las perspectivas y necesidades de la comunidad. Los alumnos participaron activamente de la territorialización, conociendo la dinámica de la realidad del territorio, estrechando los lazos con el equipo particularmente con los ACS e interactuando con los usuarios. Conclusión: La experiencia mostró el papel fundamental del enfermero en el proceso de territorialización y en el desarrollo de acciones de promoción a la salud, permitiendo a los académicos de enfermería el desarrollo de miradas críticas y dirigidos a los problemas reales de la población, dotándolos de las competencias y habilidades para el trabajo en el Sistema Único de Salud y en la Atención Primaria a la Salud.

Palabras clave: Atención Primaria a la Salud, Educación Permanente, Enfermería en Salud Pública.

\section{INTRODUÇÃO}

As Diretrizes Curriculares Nacionais (DCN) do curso de Enfermagem preveem a formação de um enfermeiro generalista, humanista, crítico e reflexivo que englobe: aprender a conhecer, aprender a fazer, aprender a viver junto e aprender a ser (SAMPAIO e CADETE, 2013). Para o desenvolvimento dessas competências, a inserção do aluno na prática profissional, sob a orientação e supervisão de professororientador e/ou preceptores deve ocorrer desde o início da graduação (BENITO et al., 2012).

A diversidade de aprendizagens desenvolvidas nos ensinos teórico-práticos e nos estágios curriculares de Enfermagem, a partir da integração ensino-serviço (ESTEVES et al., 2018), é fundamental para a formação, uma vez que os acadêmicos passam em cenários gerenciais que contribui para o aprendizado da gestão de programas e do sistema de saúde; e assistenciais para apreender a produção do cuidado em saúde.

As experiências de integração ensino-serviço foram observadas no Brasil nos últimos anos como forma de ampliação e fortalecimento da formação em saúde (BATISTA et al., 2015). Essa integração compreende o trabalho coletivo entre acadêmicos e docentes dos cursos de formação na área de Ciências da Saúde com os trabalhadores dos serviços com a finalidade de assegurar a qualidade de atenção à saúde e a formação profissional. O contato prévio com a realidade do serviço favorece o conhecimento dos determinantes sociais e promove no acadêmico um olhar ampliado do processo saúde-doença (MARIN et al., 2014; FLORES et al., 2015). Como estratégia de sustentação do Sistema Único de Saúde (SUS), a Atenção Primária à Saúde (APS) deve ser a coordenadora do cuidado e organizadora da rede de atenção à saúde, por meio da Estratégia de Saúde da Família (ESF).

A ESF baseia-se na integralidade da atenção, no trabalho em equipe, priorizando a atenção da família em seu território (FERREIRA NETO, 2016), com estabelecimento de vínculo, acolhimento e desenvolvimento de ações de prevenção, promoção, tratamento, reabilitação da saúde (GARUZI et al., 2014). Portanto, a ESF busca articular os demais níveis de complexidade de atenção com a APS.

No território a equipe de saúde da família deve acolher, responsabilizar-se e resolver os problemas de saúde dos usuários utilizando relações de vínculo e capacidade de escuta para garantir a continuidade e longitudinalidade do atendimento à população adscrita e tornando o território um princípio organizador das práticas de cuidado sob o pressuposto ético (FARIA, 2014; CAMPOS et al., 2014).

A territorialização, preconizada pelo SUS, é um dos pressupostos básicos da organização do processo 
de trabalho da ESF, tornando o território um princípio organizador das práticas de cuidado sob o pressuposto ético, além de ser um princípio técnico à medida que desloca as ações para o contexto social dos usuários, em sua existência concreta, na complexidade do contexto de vida (LEMKE e SILVA, 2013).

A formação acadêmica dos profissionais da saúde, quando estruturada a partir da territorialização, avança na construção dos referenciais políticos e epistemológicos. Esta formação não se limita a orientação voltada às necessidades do serviço e sim, orientada a emancipação humana dos sujeitos (PEREIRA e RAMOS, 2013). Tal formação se mostra crítica e libertária, ela extrapola os domínios técnico-científicos das ferramentas diagnósticas, do tratamento, do prognóstico, da etiologia e da profilaxia das enfermidades e abrange todos os aspectos estruturantes das relações e das práticas nos componentes de interesse e de relevância social (BULGARELLI et al., 2013).

Nessa perspectiva, as vivências têm sido cruciais para a formação humanística dos estudantes de Enfermagem. Sendo assim, o objetivo deste artigo foi relatar a experiência dos discentes e docentes sobre o processo de territorialização em uma equipe da Estratégia de Saúde da Família (ESF) no município de Porto Velho, Rondônia, Brasil.

\section{METODOLOGIA}

Este artigo descreve as experiências vivenciadas pelos discentes e docentes a partir de atividades realizadas no campo de prática de formação no período de julho a outubro de 2018. Advém de reflexões críticas realizadas por discentes e docentes do Curso de Enfermagem da Fundação Universidade Federal de Rondônia (UNIR), juntamente com profissionais de saúde de uma equipe de saúde da família da unidade básica de saúde (UBS) Ernandes Índio, da Zona Leste do município de Porto Velho, Rondônia, acerca do processo de territorialização. Para tanto, foi realizado o diagnóstico situacional utilizando a Territorialização (KLEBA et al., 2015).

As etapas do estudo constituíram na análise de dados secundários, na observação ativa do território e entrevista com informantes-chave. Os dados secundários foram originados de informações dos relatórios sínteses do e-SUS AB, alimentado pela Equipe de Saúde da Família (ESF) "Escola de Polícia". Também foram realizadas observações do atendimento à população da UBS, bem como entrevistas com os agentes comunitários de saúde (ACS), além de contato com usuários. Dessa forma, obtivemos as primeiras impressões sobre esse território.

Após o (re)conhecimento do território aplicamos a metodologia de Círculos de Cultura para trabalhar os principais nós críticos vivenciados no processo de territorialização, visando a construção do mapa do território. Os Círculos de Cultura visam a conquista da autonomia e a dialogicidade (VÉRAS, 2015) e desenvolvem-se a partir de etapas consecutivas e interelacionadas. A investigação temática ou descoberta do universo vocabular (o conhecimento prévio do território); Codificação (questões norteadoras, palavraschave, ou nós críticos no processo de territorialização); Descodificação (seleção de temas a partir das palavras-chave; reflexão teórico-prática); Desvelamento crítico (sistematização dos temas reapresentados nos Círculos de Cultura para análise, reflexão e encaminhamentos; consolidação e socialização das propostas); e avaliação de cada Círculo (HEIDEMANN et al., 2017).

A vivência nos Círculos de Cultura possibilitou identificar necessidades, expectativas e inquietações, que haviam sido despertadas no conhecimento do território. Para poder realizar o mapa propusemos realizar três Círculos, cujas etapas se deram a partir do terceiro momento dos Círculos de Cultura, ou seja, decodificação, objetivando discutir os nós críticos que emergiram da territorialização: vulnerabilidade e construção de mapas de território, já que durante o reconhecimento do território as duas primeiras etapas do Círculo foram 'mapeadas'.

Para processos de tomada de decisão e resolução de conflitos utilizamos o método da construção de consenso, em que, cada sujeito respeita a opinião do outro. Assim, mesmo que concordando cada participante tem capacidade de ouvir, bem como falar, não se limitando, portanto, à votação, debate ou direcionamento a partir de uma autoridade (BRONSON, 2004). 
Os Círculos de Cultura tiveram em média a duração de 60 minutos cada. Para realizar a dinâmica formamos uma roda de cadeiras, o que permitiu a melhor visualização entre os membros. Contamos com o auxílio de um aparelho de Datashow, para projeção de textos selecionados pelos docentes, que enredavam a discussão dos nós críticos gerados na territorialização.

Este estudo faz parte do projeto matriz intitulado "Estudo Sobre Morbidades em Rondônia: a assistência, a formação e o ensino em saúde em discussão", aprovado pelo Comitê de Ética em Pesquisa (CEP) do NUSAU/UNIR pelo parecer 2.548.115.

\section{RESULTADOS E DISCUSSÃO}

\section{Aprendendo, Ensinando E Mapeando O Território Vivo}

A UBS em questão conta com seis Equipes de Saúde da Família (EqsF) e seis equipes de saúde bucal e seu território adscrito está dividido em 42 microáreas, sendo que $30 \%$ das microáreas estão sem ACS, ou seja, áreas consideradas "descobertas". A equipe Escola de Polícia conta com um enfermeiro, uma médica, duas técnicas de enfermagem, quatro ACS em efetivo exercício, pois dois destes estão em licença médica há mais de um ano, além da equipe de saúde bucal (odontólogo e técnico de higiene bucal).

As ações de saúde ofertadas na UBS são imunização, consultas programadas e à demanda (enfermagem, medicina e odontologia), visitas domiciliares, curativos, retirada de pontos, administração de medicamentos, nebulização, assistência pré-natal/puerperal, coleta de material para exame laboratorial, coleta de material para exame citopatológico, ações de vigilância epidemiológica, encaminhamento para exames e consultas especializadas.

Dados do e-SUS AB mostram que a população, no período de outubro de 2017 a setembro de 2018, da equipe saúde da família Escola de Polícia (EqSFEP), era composta por 2.950 usuários, sendo $54,6 \%$ do sexo feminino. Quanto a faixa etária, destacou-se a população de adultos jovens (20 a 29 anos) com 39,8\%, em seguida os adolescentes (10 a 19 anos) com 28,3\%. Quanto a cor da pele/etnia, predominou a preta com $80,1 \%$, usuários com ensino médio completo $(36,0 \%)$ e renda familiar em torno de dois salários mínimos (58\%). Ao analisar os dados dos principais agravos no território observou-se que os problemas respiratórios apresentaram a maior frequência e entre estes predominando a asma (50,0\%).

A microárea normalmente agrega 750 habitantes e constitui a unidade escalar de atuação do profissional Agente Comunitário de Saúde (ACS). Estes, cadastram as famílias, ultrapassando, às vezes, um quantitativo de usuários e famílias a mais do que o preconizado (750 pessoas por ACS), para minimizar a falta de acesso da população à unidade, em virtude das áreas descobertas e da reposição de ACS na equipe. Esses atores conhecem as principais características e nuances das famílias adscritas e, também, conhecem muitas famílias das áreas 'descobertas'. A área da equipe Escola de Polícia é um território com bolsões de 'áreas descobertas' e, pelo relatório do e-SUS, os dados sobre o território da EqSF encontramse desatualizados.

Enquanto caminhávamos nas ruas, os agentes falavam sobre as características de seus moradores e apontavam a subdivisão geográfica entre as microáreas e as equipes. Durante as caminhadas pelo território, os alunos percebiam que cada microárea possui peculiaridade a respeito de seus usuários e sobre o perfil demográfico, epidemiológico, tecnológico, político, social e cultural em permanente transformação do território.

Podemos observar que os ACS trabalham prioritariamente com a visita domiciliar (VD) que consiste no acompanhamento das condições de saúde das famílias de sua microárea e na busca ativa de situações específicas. Nas VD, os agentes cadastram os membros da família (condição para o acesso às unidades), realizam orientações de diversos tipos, oferecem informações sobre a dinâmica de funcionamento dos serviços, entre outras ações em suas microáreas, além do trabalho realizado na própria unidade (BINDA et al., 2013; GALAVOTE et al., 2013). 
Ao caminhar com os ACS vimos que na periferia dos bairros há presença de lixo nas ruas, nos córregos e no terreno das casas alguns moradores, que incineram o lixo em frente da casa, apesar de existir coleta de lixo pelo poder público. Poucas ruas sem asfalto; iluminação pública deficitária. As famílias usam poços amazonas porque a água encanada não chega com pressão suficiente para o abastecimento adequado, fossa rudimentar, esgoto a céu aberto, falta de higienização dos ambientes, grande número de animais de rua, violência, tráfico de drogas e prostituição em determinados pontos, populações carentes e ausência de unidades policiais nas áreas. Além disto, notam-se muitos bares, como também locais para venda informal de produtos alimentícios, sem supervisão/autorização da vigilância sanitária. $\mathrm{Na}$ área desta equipe há duas escolas que atende a comunidade. Uma oferece o ensino fundamental incompleto (da $1^{\underline{a}}$ à $8^{\underline{a}}$ séries) e a outra, além do ensino fundamental, oferece também o ensino médio.

A associação de moradores e igrejas desenvolvem alguns projetos sociais que interferem positivamente na realidade local de saúde. Segundo os ACS, algumas famílias não aderem aos serviços ofertados pela equipe de saúde e não utilizam a UBS porque os traficantes não permitem.

No processo de territorialização o reconhecimento dos contextos de vida das famílias e comunidade, foi fundamental e permitiu identificar as singularidades da vida social, os problemas e necessidades de saúde (GOLDSTEIN et al., 2013). Ao consideramos o território como espaço vivo e dinâmico, não podemos deixar de incorporar a questão da violência urbana como um dos possíveis problemas de adesão aos serviços de saúde. O controle de parte do território pelos traficantes domina inclusive a vida social da população, caracterizando um 'poder paralelo'. Manter esse poder de desafiar o Estado tão bem relatadas na obra Microfísica do poder (FOUCAULT, 2014), nos traz uma reflexão sobre as novas formas de organização das comunidades periféricas.

Nas discussões com a equipe foi perceptível a necessidade de estarem atentos a transmissão de algumas doenças como a leishmaniose, em virtude da presença de animais domésticos; dengue, febre amarela, Chikungunya e Zika Vírus pela presença do Aedes aegypti. As situações percebidas no território se apresentam como fatores determinantes para a ocorrência de vários agravos que acometem os moradores e diminuem sua qualidade de vida. As condições concretas de existência das classes sociais menos favorecidas interferem diretamente no adoecimento dos sujeitos e grupos sociais e colocam em questionamento a equidade em saúde.

Os alunos participaram ativamente do processo de territorialização, conhecendo a dinâmica da realidade do território, estreitando os laços com a equipe, particularmente com os ACS e interagindo com os usuários. No processo de territorialização verificamos a interação da população com os gestores das distintas unidades prestadoras de serviços de saúde (GOLDSTEIN et al., 2013). Neste contexto, a utilização do mapeamento como fonte de informação às equipes de saúde é de fundamental importância para a comunidade, na perspectiva da equidade sobre os problemas e necessidades de saúde da comunidade.

Após o reconhecimento do território passamos a discutir sobre a elaboração do mapa a partir de Círculos de Cultura que se caracteriza como um espaço dinâmico, de interação e acolhimento com foco no diálogo em que todos participam com possibilidade de ensinar e aprender (FREIRE, 2014; SAUL e SAUL, 2016).

O primeiro Círculo foi constituído pelo enfermeiro, dois técnicos de enfermagem, quatro ACS, alunos e professores e foi extremamente enriquecedor para o grupo, na medida em que permitiu a aproximação dos participantes ao tema vulnerabilidade em saúde e o conhecimento que os sujeitos tinham sobre o assunto. Neste Círculo foi refletido sobre a fragmentação da atenção e da pouca participação da equipe de saúde bucal e da médica nas visitas domiciliares, nas atividades em grupo, na comunidade e na territorialização. Para os participantes, isso dificulta o trabalho em equipe, a atenção integral às famílias vulneráveis e a discussão e atualização do mapa da área. A discussão foi enriquecedora para o grupo na medida em que permitiu os vários atores perceberem a importância do trabalho em equipe.

A literatura demonstra que a iniquidade distributiva dos médicos no Brasil aliada a alta rotatividade dos profissionais médicos na APS promove a descontinuidade das ações de saúde, caracterizando um dos principais desafios para a efetivação integral do SUS (JESUS et al., 2017) sem esquecer de mencionar que 
a maioria destes profissionais que atua na APS ainda é formado para um modelo assistencial privatista, hospitalocêntrico, fragmentado e superespecializado, levando à desvalorização política e social da APS.

$\mathrm{Na}$ continuidade do Círculo, os ACS relatavam onde se localizam os pontos de droga, de violências, usuários que necessitam de maiores cuidados e locais onde não há saneamento básico. A partir daí, construímos o mapa conceitual, representado pelo tema vulnerabilidade na saúde da família e iniciamos o processo reflexivo.

A elaboração do mapa conceitual possibilitou a descoberta dos estrangulamentos, nós críticos, perspectivas e necessidades da comunidade, emergindo de forma bastante significativa que na APS esses aspectos constituem-se em um desafio, bem como questões sociais relacionadas à fragilidade das políticas públicas de saúde, educação, lazer e segurança pública.

Pensar coletivamente sobre ambiente, saúde-doença, violência e condições concretas de existência dessa população é discutir sobre a multideterminação da vulnerabilidade que se distribui de maneira diferente, segundo os indivíduos, regiões e grupos sociais, e relaciona-se com a pobreza, com as crises econômicas e com o nível educacional.

Para enfrentar diferenciais na vulnerabilidade é necessário um amplo processo social de construção de condições de vida, que tem certamente objetivos ligados ao setor saúde, mas não se resume a ele (FRAGA et al., 2013). A omissão e/ou o recuo do poder público em seu papel de proteção social cria ciclos de reprodução de situações de opressão e esconde a dimensão coletiva da vivência das populações em contextos de produção de vulnerabilidades, assim a vulnerabilidade decorre de discriminações e desigualdades sociais (CARMO e GUIZARDI, 2018).

No segundo Círculo começamos a elaborar o mapa do território. Para isso, questionamos os ACS, enfermeiro e técnicos de enfermagem como 'fazem o mapeamento do território'. Inicialmente, observamos que o conhecimento do território é quase exclusividade dos ACS. Tanto o enfermeiro quanto as técnicas de enfermagem tinham pouco conhecimento sobre a complexidade, as especificidades e as experiências da comunidade. Os ACS informaram que mensalmente recadastram as famílias, que conhecem os principais problemas de saúde das pessoas e de suas áreas e atualizam seus "mapas" com essas informações.

Percebemos nas falas que a territorialização se associa ao delineamento geográfico da microárea no mapa e à contagem das famílias (SILVA et al., 2016; MORAES e CANÔAS, 2013). Para esses atores os dados referentes ao contexto econômico, social, cultural e epidemiológico local não precisam estar no mapa do território. Discutimos e propomos mapear o território, atentando para a capacidade comunicativa e pedagógica que os mapas podem ter no entendimento desses territórios indo além dos limites das áreas adscritas e dos problemas emergenciais que alteram a vida das pessoas. Para Goldstein et al., (2013), o mapeamento participativo se constitui no diagnóstico local para o levantamento de informações sobre a população atendida, número de visitas domiciliares entre outras ações. O mapeamento também é importante para o levantamento de informações diretamente com as equipes por meio de por exemplo oficinas para a elaboração dos mapas.

O cadastramento dos indivíduos e famílias seja nos seus pontos singulares e/ou coletivos possibilitam a demarcação de um perfil sanitário, e a partir daí, é possível identificar informações importantes como os dados positivos e negativos nos quais a comunidade adscrita está inserida (MÔRA et al., 2013). Portanto, a territorialização se constitui como uma das ferramentas básica para o planejamento local com informações basilares para a programação de ações e a gestão do território.

No terceiro Círculo, a partir do (re)conhecimento do território, os acadêmicos de enfermagem confeccionam o mapa inteligente de forma coletiva com a equipe e acompanhado pelas docentes. Para tanto, foi feita reflexão acerca das temáticas vulnerabilidade e mapa do território. Passamos, então, para a etapa da sistematização do que seria visualizado no mapa da equipe, desencadeando, assim, a possibilidade de uma reflexão individual e a construção do conhecimento compartilhado a partir do processo de territorialização, resultado da construção de consenso entre acadêmicos, docentes, ACS, enfermeiro e 
técnicos de enfermagem.

No mapa foram identificadas as residências, locais de referência como mercados, escolas, creches, padarias, pontos de ônibus, agravos ou situações de saúde que indiquem necessidade de acompanhamento da equipe de saúde, famílias em situação de vulnerabilidade, gestantes, idosos acamados/domiciliados, dentre outros. Construiu-se os Mapas Inteligentes, dotando-os de legendas para melhor compreensão por parte da EqSF e dos usuários acerca das informações constantes em tal instrumento.

O mapa inteligente expressa o território vivo e constitui-se como ferramenta de diagnóstico da saúde da comunidade, à medida que descreve esse território, permitindo, principalmente, uma melhor focalização de problemas, facilitando assim o planejamento de ações por parte, tanto do poder público, quanto da população local (FARIA, 2013).

A proposta do mapa inteligente qualifica a problematização das questões passíveis de intervenções da equipe da UBS, traz subsídios ao processo de vigilância e atenção à saúde na medida em que favorece o acompanhamento desta população do território, fazendo-se necessário ser atualizado de acordo com a mudança do perfil populacional, geográficos e ambientais (TOMASI et al., 2018).

Além disto, percebemos que a construção do mapa inteligente pelos acadêmicos de enfermagem possibilitou uma melhor interação com a equipe, principalmente com os ACS e um processo de aprendizagem significativa onde estar com o outro e reconhecer que o outro é possuidor de um saber tornase indispensável para a produção do consenso. Nessa perspectiva, podemos dizer que houve o compartilhamento entre os saberes "populares" e os "científicos".

$\mathrm{Na}$ avaliação dos Círculos ficou clara a importância de integração ensino-serviço, bem como trabalhar com a construção de consenso, o que pode fomentar a transformação dos processos formativos dos enfermeiros. A participação no processo de territorialização da equipe Escola de Polícia possibilitou vivências intensas, algumas desconfortáveis, outras mobilizadoras de afetos positivos que os estudantes afirmaram ter repercutido em seus processos de formação profissional e também pessoal.

\section{CONSIDERAÇÕES FINAIS}

A existência de diversas configurações de famílias e perfis demográfico, epidemiológico, tecnológico, político, social e cultural em permanente transformação, nos permitiu apreender o território vivo, a existência de grupos e subgrupos, que, de outra forma, não se evidenciariam atingindo então uma melhor forma de compreensão das relações ali existentes. O diálogo promovido no desenvolvimento dos Círculos de Cultura sobre os nós críticos do território possibilitou um espaço dialógico e participativo. Desse modo, resultou na construção do mapa do território, contribuindo para que todos os participantes ressignificassem suas compreensões a respeito da territorialização. A experiência mostrou o papel fundamental do enfermeiro no processo de territorialização e no desenvolvimento de ações sanitárias, permitindo aos acadêmicos de enfermagem o desenvolvimento de olhares críticos e voltados para os problemas reais da população, dotando-os das competências e habilidades para o trabalho no SUS e na APS que são assistenciais, de gestão, de pesquisa e de educação permanente.

\section{REFERÊNCIAS}

1. BATISTA SHSS, JANSEN B, ASSIS EQ et al. Formação em saúde: reflexões a partir dos programas Pró-Saúde e Pet-Saúde. Interface - Comum Saúde Educ, 2015; 19(supl. 1):743-52.

2. BENITO GAV, TRISTÃO KM, PAULA ACSF et al. Desenvolvimento de competências gerais durante o estágio supervisionado. Rev Bras Enferm. 2012; 65(1): 172-8.

3. BINDA JB, BIANCO MF, SOUSA EM. O trabalho dos agentes comunitários de saúde em evidência: uma análise com foco na atividade. Saúde Socied. 2013; 22(2):389-402.

4. BRONSON JN. Focalização, sinergia \& construção de consenso para projetos temáticos. 2004. 
5. BULGARELLI AF, SOUZA KR, BAUMGARTEN A et al. Formação em saúde com vivência no Sistema Único de Saúde (SUS): percepções de estudantes do curso de Odontologia da Universidade Federal do Rio Grande do Sul (UFRGS), Brasil. Interface - Comum Saúde Educ. 2013.

6. CAMPOS RTO, FERRER AL, GAMA CAP et al. Avaliação da qualidade do acesso na atenção primária de uma grande cidade brasileira na perspectiva dos usuários. Saúde Debate. 2014; 38(Especial):252-64.

7. CARMO ME, GUIZARDI FL. O conceito de vulnerabilidade e seus sentidos para as políticas públicas de saúde e assistência social. Cad. Saúde Pública. 2018; 34(3) :e00101417.

8. ESTEVES LSF, CUNHA ICKO, BOHOMOL E et al. O estágio curricular supervisionado na graduação em enfermagem: revisão integrativa. Rev Bras Enferm., Brasília, 2018; v.71, n. suppl 4, p.1842-53.

9. FARIA RM. A territorialização da atenção primária à saúde no Sistema Único de Saúde e a construção de uma perspectiva de adequação dos serviços aos perfis do território. Hygeia. 2013; 9(16):131-47.

10. FARIA RSR. Acesso no contexto da ESF em um município do Vale Jequitinhonha - MG. 2014. 128f/ Dissertação (Mestrado). Universidade Federal de Minas Gerais, Escola de Enfermagem, Belo Horizonte, 2014.

11. FERREIRA NETO JL, OLIVEIRA GL, VIANA NO et al. Integralidade, condições de oferta de serviços e processo de trabalho de Equipes de Saúde da Família em Belo Horizonte. Saúde Debate. 2016; 40(111):179-92.

12. FLORES LM, TRINDADE AL, LORETO DR et al. Avaliação do Programa de Educação pelo Trabalho para Saúde PET-Saúde/Vigilância em Saúde pelos seus atores. Interface - Comum Saúde Educ. 2015; 19(Supl 1):923-30.

13. FOUCAULT M. Microfísica do poder. 28. Ed. São Paulo: Paz e Terra, 2014.

14. FRAGA AB, LOPES ACS, PINTO ALS et al. Ministério da Saúde, Secretaria de Vigilância em Saúde. Curso de extensão em promoção da saúde para gestores do SUS com enfoque no Programa Academia da Saúde. Brasília: Fundação Universidade de Brasília: CEAD, 2013.

15. FREIRE P. Educação como prática da liberdade. Rio de Janeiro: Paz e Terra, 2014.

16. GALAVOTE HS, FRANCO TB, LIMA RCD et al. Alegrias e tristezas no cotidiano de trabalho do agente comunitário de saúde: cenários de paixões e afetamentos. Interface Comum Saúde Educ. 2013; 17(46):575-86.

17. GARUZI M, ACHITTI MCO, SATO CA et al. Acolhimento na Estratégia Saúde da Família: revisão integrativa. Rev Panam de Salud Publica. 2014; 35(2):144-9.

18. GOLDSTEIN RA, BARCELLOS C, MAGALHÃES MAF et al. A experiência de mapeamento participativo para a construção de uma alternativa cartográfica para a ESF. Ciênc Saúde Coletiva. 2013; 18(1):45-56.

19. HEIDEMANN ITSB, DALMOLIN IS, RUMOR PCF et al. Reflexões sobre o itinerário de pesquisa de Paulo Freire: contribuições para a saúde. Texto Contexto Enferm. 2017; 26(4): e0680017.

20. JESUS RA, MEDINA MG, PRADO NMBL. Programa Mais Médicos: análise documental dos eventos críticos e posicionamento dos atores sociais. Interface - Comum Saúde Educ. 2017; 21(Supl.1):1241-55.

21. KLEBA ME, DUARTE TF, ROMANINI A et al. Estimativa rápida participativa como ferramenta de diagnóstico na estratégia saúde da família. REVISTA GRIFOS. 2015; (38/39):159-71.

22. LEMKE RA, SILVA RAN. Itinerários de construção de uma lógica territorial do cuidado. Psicol. Soc. 2013; 25(Supl. 2):9-20.

23. MARIN MJS, OLIVEIRA MAC, OTANI MAP et al. A integração ensino-serviço na formação de enfermeiros e médicos: a experiência da FAMEMA. Ciênc. Saúde Coletiva. 2014; 19(3):967-74.

24. MÔRA LB, GOMES JC, MORAES MLS et.al. O uso da territorialização para apoio ao planejamento das ações de uma unidade de Saúde da Família. Belo Horizonte: $2^{\circ}$ Congresso brasileiro de política, planejamento e gestão em saúde, 2013.

25. MORAES DE, CANÔAS SS. O conceito de "território" e seu significado no campo da atenção primária a saúde. Revista Desenvolvimento Social. 2013; 1(9):49-57.

26. PEREIRA IB, RAMOS MN. Educação profissional em saúde. 2ed. Rio de Janeiro: Editora Fiocruz; 2013.

27. SAMPAIO FC, CADETE MMM. A formação de enfermeiro na visão dos acadêmicos de enfermagem: atividades respaldadas na problematização. J Nurs UFPE. 2013; 7(3):657-64.

28. SAUL AM, SAUL A. Paulo Freire's contributions to teacher education: foundations and practices of a counterhegemonic paradigm. Educar em Revista. 2016; 61:19-35.

29. SILVA LCT, PASQUAL KK, CHIARI MF et al. Estratégia saúde da família e território: percepção dos trabalhadores da atenção básica em saúde. Investigação Qualitativa em Saúde. 2016; 2:1622-31.

30. TOMASI YT, SOUZA JB, MADUREIRA VSF. Diagnóstico comunitário na estratégia saúde da família: potencialidades e desafios. Rev enferm UFPE on line. 2018; 12(6):1546-53.

31. VÉRAS GCB, SOUZA MM, TEOTÔNIO LF et al. Círculos de Cultura e Qualificação Profissional para Agentes Comunitários de Saúde da Unidade Básica de Saúde São José/PAPS - Relato de Experiência. Revista Brasileira de Extensão Universitária. 2015; 6(1):53-8. 